\title{
The safety and efficacy of tPA intravenous thrombolysis for treating acute ischemic stroke patients with a history of cerebral hemorrhage
}

\author{
Guang-jian Zhao ${ }^{1}$, Zi-ran Wang ${ }^{1}$, Fan-zhen Lin $^{2}$, Yan-sen Cui ${ }^{1}$ and Shun-liang $\mathrm{Xu}^{3}$ \\ ${ }^{1}$ Department of Neurology, Linyi People's Hospital Affiliated to Shandong University, Linyi, Shandong, China \\ ${ }^{2}$ General Medicine, Linyi People's Hospital Affiliated to Shandong University, Linyi, Shandong, China \\ ${ }^{3}$ Department of Neurology, The Second Hospital of Shandong University, Jinan, Shandong, China
}

\begin{abstract}
Alteplase (tPA) intravenous thrombolysis is an effective treatment for acute ischemic stroke (AIS) when administered within $4.5 \mathrm{~h}$ of initial stroke symptoms. Here, its safety and efficacy were evaluated among AIS patients with a previous history of cerebral hemorrhage. Patients who arrived at the hospital within $4.5 \mathrm{~h}$ of initial stroke symptoms and who were treated with tPA intravenous thrombolysis or conventional therapies were analyzed. The 90 -day modified Rankin scale (90-d mRS) was used alongside mortality and incidence of symptomatic intracerebral hemorrhage $(\mathrm{SICH})$ rates to evaluate the curative effect of these therapies. Among 1,694 AIS patients, 805 patients were treated with intravenous thrombolysis, including patients with $(n=793)$ or without $(n=12)$ a history of cerebral hemorrhage, and the rate of incidence of SICH significantly differed between them ( 8.3 vs $4.3 \%, P=0.039$ ). No significant difference was found in $90-\mathrm{d}$ mRS measurements (41.7 vs $43.6 \%, P=0.530)$ and $90-d$ mortality rates $(8.3$ vs $6.5 \%, P=0.946)$. A total of 76 AIS patients with a history of cerebral hemorrhage received tPA thrombolytic therapy $(n=12)$ or conventional therapy $(n=64)$, and a significant difference was noted in the $90-d$ mRS scores between the two groups (41.7 vs $23.4 \%, P=0.029)$, while no significant difference was found in $\mathrm{SICH}$ measurements (8.3 vs $4.6 \%, \mathrm{P}=0.610)$ and $90-\mathrm{d}$ mortality rates $(8.3$ vs $9.4 \%, \mathrm{P}=0.227$ ). A history of cerebral hemorrhage is not an absolute contraindication for thrombolytic therapy; tPA intravenous thrombolysis does not increase $\mathrm{SICH}$ measurements and mortality rates in patients with a history of cerebral hemorrhage, and they may benefit from thrombolytic therapy.
\end{abstract}

Key words: tPA; Acute ischemic stroke; History of cerebral hemorrhage; Intravenous thrombolysis; Contraindication

\section{Introduction}

Acute ischemic stroke (AIS) is a major cause of death and disability in the United States (1). Hypertension is a common risk factor for both ischemic and hemorrhagic strokes (2), and incidence of hypertensive intracerebral hemorrhage in the Asian population is higher than in Europe and the United States due to racial and dietary habits (3). The use of alteplase (tPA) intravenous thrombolysis within $4.5 \mathrm{~h}$ of symptom onset during stroke is the only therapy that reduces disability among AIS patients (4).

Early clinical trials of intravenous thrombolytic therapy for AIS have primarily been designed to avoid increased symptomatic intracerebral hemorrhage $(\mathrm{SICH})$, mortality rates, and negative treatment outcomes (5). Currently, the stroke guidelines of every country classify a history of intracranial hemorrhage as a contraindication for intravenous thrombolytic therapy $(6,7)$. Thus, patients with a history of cerebral hemorrhage cannot receive intravenous thrombolysis, regardless of the location and amount of bleeding or recovery situation.

Contraindication in stroke guidelines is based on contraindications from previous randomized controlled clinical trials, which lack rigorous clinical evidence (8). Recent studies have shown that contraindications for intravenous thrombolysis are too stringent and that some patients do not receive effective treatment for stroke because of this. In fact, the use of intravenous thrombolysis for AIS is supported by an increasing number of studies $(9,10)$, while contraindications continue to be shown as unnecessary through clinical experience (11). However, the ability of AIS patients with a history of cerebral hemorrhage to accept tPA thrombolytic therapy remains in question, and the safety of this treatment requires further evaluation. Thus, the present study evaluated the safety and efficacy of intravenous thrombolysis

Correspondence: Shun-liang Xu: <shunliangxu@126.com>

Received June 21, 2018 | Accepted November 19, 2018 
for AIS patients with a previous history of cerebral hemorrhage.

\section{Material and Methods}

\section{Patients}

This retrospective study was approved by the Ethics Committee of the Linyi People's Hospital Affiliated to Shandong University and followed the ethical principles stated in the Declaration of Helsinki and the relevant laws of the People's Republic of China. Written informed consent was obtained from all patients.

The study retrospectively analyzed patients with AIS who were treated in Linyi People's Hospital Affiliated to Shandong University from July 2015 to December 2017. The use rate of tPA (Boehringer Ingelheim, $\mathrm{GmbH}$ ) intravenous thrombolytic therapy and the treatment situations of AIS patients with previous histories of cerebral hemorrhage within $4.5 \mathrm{~h}$ of arrival at the hospital were obtained. Patients who underwent bridge therapy were not included in this study. All patients treated with tPA thrombolysis had undergone laboratory tests, head imaging, and were measured according to the National Institutes of Health Stroke Scale (NIHSS) before thrombolysis. Within $24 \mathrm{~h}$ of tPA thrombolysis treatment, all patients received necessary nursing supervision, such as electrocardiograph monitoring. When cerebral hemorrhage was controlled at $24 \mathrm{~h}$, patients received antithrombotic treatment and other symptomatic treatments for certain risk factors. Follow-up head imaging was performed 24-36 $\mathrm{h}$ after the onset of stroke, followed by NIHSS scoring, and a modified Rankin scale (mRS) score was taken with a 90-day ( $\pm 7 \mathrm{~d})$ followup after the onset of stroke.

\section{Patients with a history of cerebral hemorrhage}

History of cerebral hemorrhage is defined as a definite history of spontaneous cerebral hemorrhage confirmed by previous cerebral computed tomography. However, subdural hematomas caused by trauma and subarachnoid hemorrhages were excluded from this study. For safety, patients with a history of cerebral hemorrhage were treated with a low dose of tPA $(0.6 \mathrm{mg} / \mathrm{kg})$.

\section{Evaluation of safety and effectiveness}

The main safety outcome measures were $\mathrm{SICH}$ after thrombolysis and the mortality rate of patients at 90 days after the onset of stroke. Based on the research standards of the European Cooperative Acute Stroke Study II (9), $\mathrm{SICH}$ is defined as bleeding indicated by head image examination after thrombolytic therapy and a NIHSS score of 4 points or more than the baseline NIHSS score or than the baseline NIHSS score for 24-36 $\mathrm{h}$ after thrombolysis. The primary effective outcome was measured using the 90-d mRS score after thrombolysis (10), with a score of 0-1 indicating a good clinical outcome, 2-5 indicating varying degrees of dysfunction, and 6 indicating death.

\section{Statistical analysis}

The SPSS 22.0 software package was used for statistical analysis. The measurement data are reported as a median and four quantile intervals, and the difference between groups was examined using a Mann-Whitney $\mathrm{U}$ test. Count data are reported as frequency and percentage, and a $\chi^{2}$ test was used to analyze the difference between the two treatment groups. The odds ratio (OR) and the $95 \%$ confidence interval $(\mathrm{Cl})$ for safety and effectiveness outcomes and the modified OR and its $95 \% \mathrm{Cl}$ were compared by univariate and multivariate regression analyses, with $\mathrm{P}<0.05$ being statistically significant.

\section{Results}

\section{General data and baseline characteristics}

The study was a retrospective analysis of 17,285 patients with AIS admitted to Linyi People's Hospital from July 2015 to December 2017, with 1,694 patients arriving at the hospital emergency department within $4.5 \mathrm{~h}$ of the onset of stroke. Among these patients, 1,022 received t-PA intravenous thrombolysis, and 130 cases were excluded due to bridge therapy and 87 cases due to incomplete data or lost to follow-up. Thus, 805 patients with AIS received the t-PA intravenous thrombolysis were enrolled in this study, including 793 patients without a previous history of cerebral hemorrhage. There were 76 AIS patients with a history of cerebral hemorrhage, including 12 patients who received tPA thrombolytic therapy, and 64 patients who received conventional treatments (Figure 1).

Among the patients who received tPA intravenous thrombolytic therapy, differences in patients who received a median tPA dose (0.6 vs $0.79 \mathrm{mg} / \mathrm{kg}, \mathrm{P}=0.003)$ and who had a history of hypertension (83.3 vs $62.0 \%, \mathrm{P}=0.028$ ) and drinking (50 vs $30.5 \%, \mathrm{P}=0.039$ ) were significant for patients with and without a history of cerebral hemorrhage. There was no significant difference in other baseline characteristics, such as age, gender, NIHSS score, mRS score of $0-1$ before stroke, history of diabetes mellitus, or atrial fibrillation (Table 1).

In patients with a previous history of cerebral hemorrhage, in addition to mRS scores of 0-1 before stroke (91.7 vs $78.1 \%, P=0.022$ ), the time interval of cerebral hemorrhage occurrence ( 6.5 vs 3 years, $\mathrm{P}=0.003$ ) was markedly different among patients who received tPA thrombolytic therapy or conventional therapy. There was no significant difference in other baseline features, including age, gender, NIHSS score, median tPA dose, and history of diabetes mellitus or atrial fibrillation (Table 2).

\section{Safety and effectiveness of intravenous thrombolysis}

In patients who received tPA intravenous thrombolytic therapy, $\mathrm{SICH}$ (8.3 vs $4.3 \%, \mathrm{P}=0.039$ ) results were significantly different compared to results from patients with or without a history of cerebral hemorrhage, while no significant difference was observed for $90-\mathrm{d}$ mRS scores 


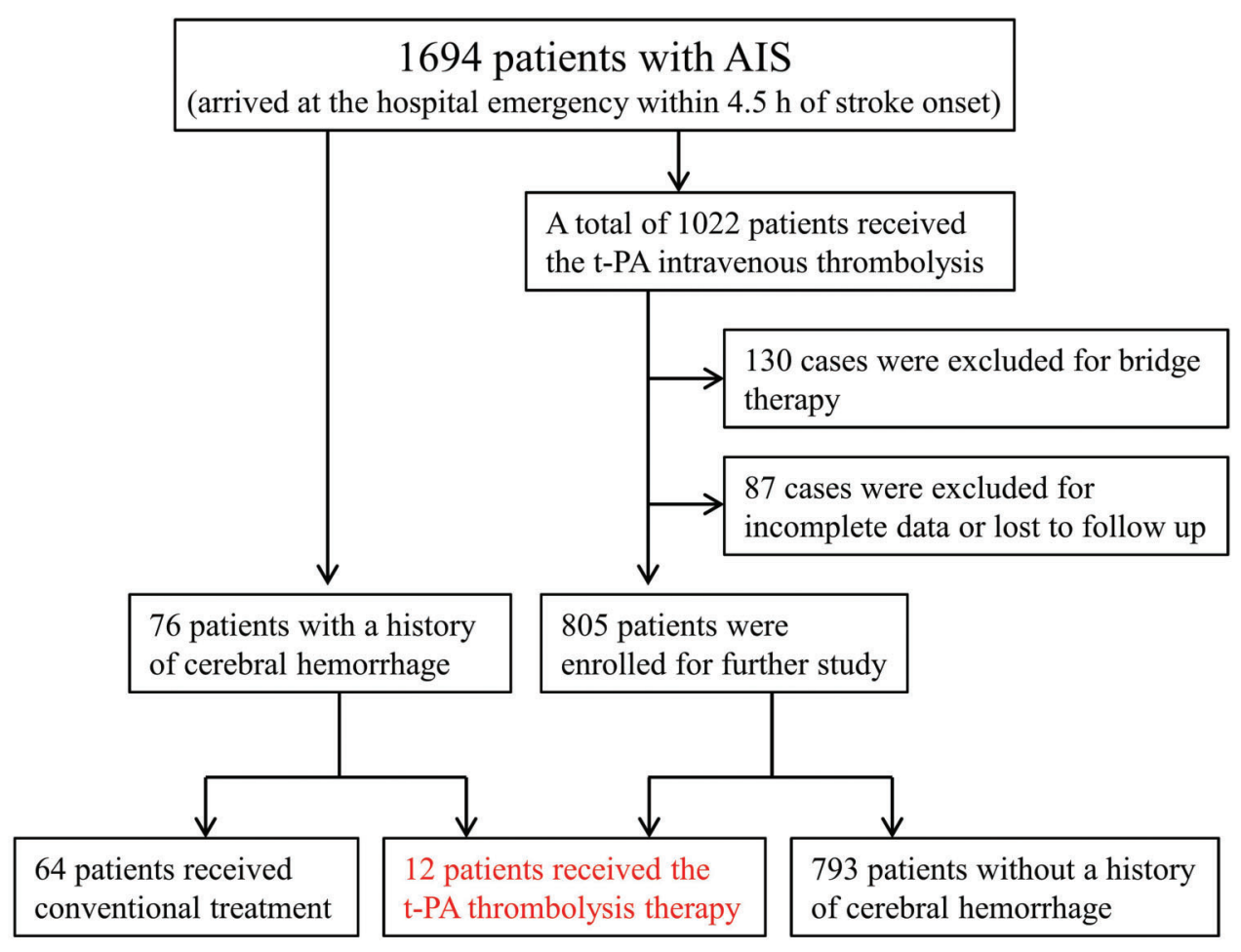

Figure 1. Patient flowchart: Among 1,694 acute ischemic stroke (AIS) patients, 805 patients received the alteplase (t-PA) intravenous thrombolysis were enrolled in the study, including 793 patients without a history of cerebral hemorrhage. In addition, 76 AIS patients had a history of cerebral hemorrhage, including 12 patients who received tPA thrombolytic therapy and 64 patients who received conventional treatment.

of $0-1$ (41.7 vs $43.6 \%, P=0.530)$ and $90-d$ mortality rate (8.3 vs $6.5 \%, \mathrm{P}=0.946$ ) (Table 3 ). In patients with a previous history of cerebral hemorrhage, a $90-\mathrm{d} \mathrm{mRS}$ score of 0-1 (41.7 vs $23.4 \%, P=0.029)$ was significantly different between those who received tPA thrombolytic therapy and those who received conventional therapy, although no significant difference was noted in $\mathrm{SICH}$ (8.3 vs $4.6 \%$, $\mathrm{P}=0.610$ ) and 90 -d mortality rate (8.3 vs $9.4 \%, \mathrm{P}=0.227$ ) (Table 4). Figure 2 presents the mRS scores of the three groups and scores at the 90-d follow-up.

\section{Discussion}

In this study, 1,694 AIS patients arrived at the hospital emergency department within $4.5 \mathrm{~h}$ of the onset of stroke, and $76(4.5 \%)$ patients had a previous history of cerebral hemorrhage. Based on the contraindication in stroke guidelines, those 76 patients should not receive intravenous thrombolysis, regardless of the time interval of cerebral hemorrhage occurrence, bleeding site, bleeding volume, and recovery condition of cerebral hemorrhage. According to their family's wishes, for some patients who had a cerebral hemorrhage long before and an optimal recovery, tPA intravenous thrombolysis was used at a median tPA dose of $0.64 \mathrm{mg} / \mathrm{kg}$ ( vs $0.79 \mathrm{P}=0.003$ for patients without a history of cerebral hemorrhage). For safety, patients with a history of cerebral hemorrhage were treated with a low dose of tPA $(0.6 \mathrm{mg} / \mathrm{kg})$ thrombolytic therapy. A significant difference was observed among patients with a history of hypertension (83.3 vs $62.0 \%$, $\mathrm{P}=0.028)$ and drinking ( 50 vs $30.5 \%, \mathrm{P}=0.039)$ in the two groups. Hypertension may be the most important risk factor for cerebral hemorrhage, and the interaction between hypertension and alcohol in stroke is complicated (12-14). No significant difference was found in other baseline characteristics.

No significant difference in the $90-\mathrm{d}$ mRS score of $0-1$ (41.7 vs 43.6\%, $P=0.530)$ and the 90 - $d$ mortality rate (8.3 vs $6.5 \%, \mathrm{P}=0.946$ ) was noted, suggesting that both groups experienced similar treatment effects, without an increase in the mortality rate. However, a significant difference in $\mathrm{SICH}$ (8.3 vs $4.3 \%, \mathrm{P}=0.039$ ) between the groups suggests a higher risk of bleeding in patients with a history of cerebral hemorrhage after treatment with IPA intravenous thrombolysis, although this could be a result of bias due to the small number of patients with a previous 
Table 1. Demographic and baseline clinical data for patients undergoing intravenous thrombolysis.

\begin{tabular}{|c|c|c|c|}
\hline Variables & $\begin{array}{l}\text { With previous } \\
\text { intracerebral hemorrhage } \\
\qquad(n=12)\end{array}$ & $\begin{array}{l}\text { Without previous } \\
\text { intracerebral hemorrhage } \\
\qquad(n=793)\end{array}$ & $P$ value \\
\hline \multicolumn{4}{|l|}{ Demographic factors } \\
\hline Age (year; M, IQR) & $66(60-73)$ & $66(60-72)$ & 0.316 \\
\hline Male $(n, \%)$ & $7(58.3)$ & $490(61.8)$ & 0.835 \\
\hline Median tPA dose $(\mathrm{mg} / \mathrm{kg})$ & $0.64(0.60-0.69)$ & $0.79(0.60-0.90)$ & 0.003 \\
\hline Before stroke mRS 0-1scores (n, \%) & $11(91.7)$ & $737(94.5)$ & 0.611 \\
\hline \multicolumn{4}{|l|}{ Vascular risk factors $(\mathrm{n}, \%)$} \\
\hline Hypertension & $10(83.3)$ & $492(62.0)$ & 0.028 \\
\hline Hyperlipidemia & $4(33.3)$ & $301(37.9)$ & 0.837 \\
\hline Diabetes & $2(16.7)$ & $153(19.3)$ & 0.561 \\
\hline Ischemic heart disease & $5(41.7)$ & $260(32.8)$ & 0.157 \\
\hline Atrial fibrillation & $3(25.0)$ & $191(24.1)$ & 0.486 \\
\hline Smoking & $5(41.7)$ & $350(44.1)$ & 0.302 \\
\hline Drinking & $6(50.0)$ & $242(30.5)$ & 0.039 \\
\hline \multicolumn{4}{|l|}{ Stroke etiology typing (n, \%) } \\
\hline Atherosclerosis & $5(41.7)$ & $305(38.5)$ & 0.809 \\
\hline Arteriolar occlusion & $4(33.3)$ & $270(34.0)$ & 0.761 \\
\hline Cardiogenic embolism & $2(16.7)$ & $176(22.2)$ & 0.297 \\
\hline Other determined causes & $1(8.3)$ & $23(2.9)$ & 0.180 \\
\hline Undetermined etiology & $0(0)$ & $19(2.4)$ & 1.0 \\
\hline Baseline NIHSS score (M, IQR) & $13(10-16)$ & $12(10-15)$ & 0.483 \\
\hline Infarction located in the anterior circulation (n, \%) & $9(75.0)$ & $629(79.3)$ & 0.584 \\
\hline
\end{tabular}

M: median; IQR: interquartile range; tPA: alteplase; mRS: modified Rankin scale; NIHSS: National Institutes of Health Stroke Scale. Statistical analysis was done with Mann-Whitney $U$ test and $\chi^{2}$ test.

history of cerebral hemorrhage who received thrombolytic therapy in this study. The mortality rate did not increase in patients after tPA intravenous thrombolysis, implying that they may benefit from this therapy.

Lee et al. (15) performed a retrospective analysis of 1,495 cases of intravenous thrombolysis, 73 of which included a history of cerebral hemorrhage (4.9\%); incidence of $\mathrm{SICH}$ was 6.8 vs $4.6 \%$ in patients with or without a history of cerebral hemorrhage after thrombolysis, respectively, and no significant difference was found between them. AbdelRazek et al. (16) suggested that patients with a history of cerebral hemorrhage who received thrombolytic therapy had no increased risk of $\mathrm{SICH}$. This conclusion was based on a retrospective analysis of brain imaging data from 640 patients admitted to a hospital between January 2006 and April 2014, who received tPA intravenous thrombolysis and who had a history of cerebral hemorrhage prior to receiving thrombolysis. Twenty-seven of these patients had experienced previous cerebral hemorrhage and only $1(3.7 \%)$ patient presented $\mathrm{SICH}$ after tPA intravenous thrombolysis. Twenty-five cases $(4.1 \%)$ presented $\mathrm{SICH}$ among the remaining 613 patients who received thrombolytic therapy. Meretoja et al. (17) studied 985 AIS patients who were eligible for treatment with alteplase but received thrombolytic therapy: 1 patient had a previous a history of cerebral hemorrhage and 2 patients had a history of subarachnoid hemorrhage. No $\mathrm{SICH}$ occurred after thrombolysis, although 2 patients had $90-d$ mRS scores of $0-1$, and 1 patient had an mRS score of 3 . These results suggest that a history of cerebral hemorrhage is not an absolute contraindication for thrombolytic therapy and that tPA intravenous thrombolysis does not increase the incidence of $\mathrm{SICH}$ and mortality rates among recipients.

The current study found a significant difference among patients with a previous history of cerebral hemorrhage who received IPA thrombolytic therapy and conventional therapy in terms of $90-\mathrm{d} \mathrm{mRS}$ scores of $0-1$ (41.7 vs $23.4 \%, P=0.029$, respectively), while no significant difference was found in incidence of $\mathrm{SICH}$ (8.3 vs 4.6\%, $\mathrm{P}=0.610$, respectively) or $90-\mathrm{d}$ mortality (8.3 vs $9.4 \%$, $\mathrm{P}=0.227$, respectively). These findings demonstrate that tPA intravenous thrombolysis provides a better prognosis than conventional treatment for patients with a previous history of cerebral hemorrhage, with no significant difference in $\mathrm{SICH}$, mortality, and safety. Based on safety considerations, when administering thrombolytic therapy, physicians should take into account patient history, particularly the 
Table 2. Demographic and baseline clinical data of patients with previous intracerebral hemorrhage.

\begin{tabular}{|c|c|c|c|}
\hline Variables & Intravenous thrombolysis $(n=12)$ & Conventional therapy $(n=64)$ & $P$ value \\
\hline \multicolumn{4}{|l|}{ Demographic factors } \\
\hline Age (year; M, IQR) & $66(60-73)$ & $67(58-72)$ & 0.185 \\
\hline Male $(n, \%)$ & $7(58.3)$ & $39(61.0)$ & 0.291 \\
\hline Before stroke mRS (0-1) (n, \%) & $11(91.7)$ & $50(78.1)$ & 0.022 \\
\hline \multicolumn{4}{|l|}{ Vascular risk factors $(\mathrm{n}, \%)$} \\
\hline Hypertension & $10(83.3)$ & $53(82.8)$ & 0.625 \\
\hline Hyperlipidemia & $4(33.3)$ & $24(37.5)$ & 0.334 \\
\hline Diabetes & $2(16.7)$ & $13(20.3)$ & 0.513 \\
\hline Ischemic heart disease & $5(41.7)$ & $25(39.1)$ & 0.610 \\
\hline Atrial fibrillation & $3(25.0)$ & $15(23.4)$ & 0.495 \\
\hline Smoking & $5(41.7)$ & $28(43.8)$ & 0.121 \\
\hline Drinking & $6(50.0)$ & $33(51.6)$ & 0.364 \\
\hline Time interval of cerebral hemorrhage occurrence (year) & $6.5(5-8.75)$ & $3(2-6)$ & 0.007 \\
\hline \multicolumn{4}{|l|}{ Stroke etiology typing $(\mathrm{n}, \%)$} \\
\hline Atherosclerosis & $5(41.7)$ & $27(42.2)$ & 0.156 \\
\hline Arteriolar occlusion & $4(33.3)$ & $21(32.8)$ & 0.512 \\
\hline Cardiogenic embolism & $2(16.7)$ & $13(20.3)$ & 0.249 \\
\hline Other determined causes & $1(8.3)$ & $3(4.7)$ & 0.655 \\
\hline Undetermined etiology & $0(0)$ & $1(1.6)$ & 1.0 \\
\hline Baseline NIHSS score (M, IQR) & $13(10-16)$ & $14(11-16)$ & 0.196 \\
\hline Infarction located in the anterior circulation (n, \%) & $9(75.0)$ & $49(76.5)$ & 0.362 \\
\hline
\end{tabular}

M: media; IQR: interquartile range; mRS: modified Rankin scale; NIHSS: National Institutes of Health Stroke Scale. Statistical analysis was done with Mann-Whitney $U$ test and $\chi^{2}$ test.

length of cerebral hemorrhage and the recovery condition. Choosing patients who previously experienced better recovery leads to better $\mathrm{mRS}$ scores of $0-1$ (91.7 vs $78.1 \%, P=0.022)$ and who have no medical history of cerebral hemorrhage (6.5 vs 3 years, $P=0.003)$ results in significant differences among patients treated with thrombolytic therapy and those with conventional therapy. This indicates that TPA intravenous thrombolysis is safe and effective for some AIS patients with previous cerebral hemorrhage of a long duration but who had experienced positive recovery.

Taking into account the potential risk of $\mathrm{SICH}$ after thrombolysis, all AIS patients with a history of cerebral hemorrhage should be given low doses of intravenous thrombolysis (median tPA dose $=0.6 \mathrm{mg} / \mathrm{kg}$ ). Japan Alteplase Clinical Trial (J-ACT) research results suggest that a low dose of tPA $(0.6 \mathrm{mg} / \mathrm{kg})$ intravenous thrombolytic therapy in the Japanese population can obtain similar clinical efficacy as the standard dose of National Institute of Neurological Disorders and Stroke (NINDS) and can reduce the risk of $\mathrm{SICH}(18)$. Although the J-ACT study did not establish a control group, the subsequent J-ACT II (19) and J-MARS studies (20) showed the safety and efficacy of intravenous thrombolytic therapy with low-dose tPA $(0.6 \mathrm{mg} / \mathrm{kg})$. The results of enhanced control in a hypertension and thrombolysis stroke study showed that low-dose
tPA led to a lower risk for intracranial hemorrhage compared to the standard dose $(0.9 \mathrm{mg} / \mathrm{kg})$ and that low dose tPA reduces mortality rates (21). Thus, low-dose tPA thrombolysis is suggested to reduce the risk for intracranial hemorrhage among patients at higher risk for the condition when treated with thrombolysis.

This study was limited by being retrospective rather than prospective. Additionally, the number of patients included in the two treatment groups was not adequately balanced, especially for patients with a history of cerebral hemorrhage who were treated with intravenous thrombolytic therapy, which may have affected the results. Patients with this history were not randomly divided, and as a result, patients at a low risk for bleeding received intravenous thrombolytic therapy, which might also have affected the results. Finally, because cerebral hemorrhage occurred long before among patients treated with intravenous thrombolysis, imaging data had been lost; thus, bleeding volume was unclear. Thus, the relationship between amount of bleeding and risk related to IPA intravenous thrombolysis could not be determined.

In summary, these results suggest that a history of cerebral hemorrhage is not an absolute contraindication for thrombolytic therapy. In some patients, tPA intravenous thrombolysis does not increase the risk of $\mathrm{SICH}$ or mortality, and these patients may benefit from intravenous 
Table 3. Comparison of the efficacy and safety of intravenous thrombolysis in patients.

\begin{tabular}{|c|c|c|c|c|c|}
\hline Outcomes & $\begin{array}{l}\text { With previous } \\
\text { intracerebral hemorrhage } \\
\qquad(n=12)\end{array}$ & $\begin{array}{c}\text { Without previous } \\
\text { intracerebral hemorrhage } \\
\qquad(n=793)\end{array}$ & OR $(95 \% \mathrm{Cl})$ & $\chi^{2}$ & $P$ value \\
\hline 90-d mRS score $(0-1)(n, \%)$ & $5(41.7)$ & $346(43.6)$ & $0.539(0.146-2.538)$ & 0.527 & 0.530 \\
\hline Symptomatic cerebral hemorrhage & $1(8.3)$ & $34(4.3)$ & $0.072(0.0013-0.917)$ & 3.912 & 0.048 \\
\hline 90-d mortality $(\mathrm{n}, \%)$ & $1(8.3)$ & $52(6.5)$ & $0.837(0.068-1.416)$ & 0.028 & 0.946 \\
\hline
\end{tabular}

OR: odds ratio; Cl: confidence interval; mRS: modified Rankin scale.

Table 4. Comparison of the efficacy and safety of intravenous thrombolysis among patients with previous intracerebral hemorrhage.

\begin{tabular}{|c|c|c|c|c|c|}
\hline Outcomes & $\begin{array}{l}\text { Intravenous thrombolysis } \\
\qquad(\mathrm{n}=12)\end{array}$ & $\begin{array}{l}\text { Conventional therapy } \\
\qquad(\mathrm{n}=64)\end{array}$ & OR $(95 \% \mathrm{Cl})$ & $\chi^{2}$ & $P$ value \\
\hline 90-d mRS score (0-1) (n, \%) & $5(41.7)$ & $15(23.4)$ & $0.172(0.035-0.907)$ & 4.305 & 0.029 \\
\hline Symptomatic cerebral hemorrhage & $1(8.3)$ & $3(4.6)$ & $0.351(0.153-0.708)$ & 0.518 & 0.610 \\
\hline 90-d mortality $(n, \%)$ & $1(8.3)$ & $6(9.4)$ & $0.785(0.339-1.354)$ & 1.462 & 0.227 \\
\hline
\end{tabular}

OR: odds ratio; Cl: confidence interval; mRS: modified Rankin scale.

mRS score

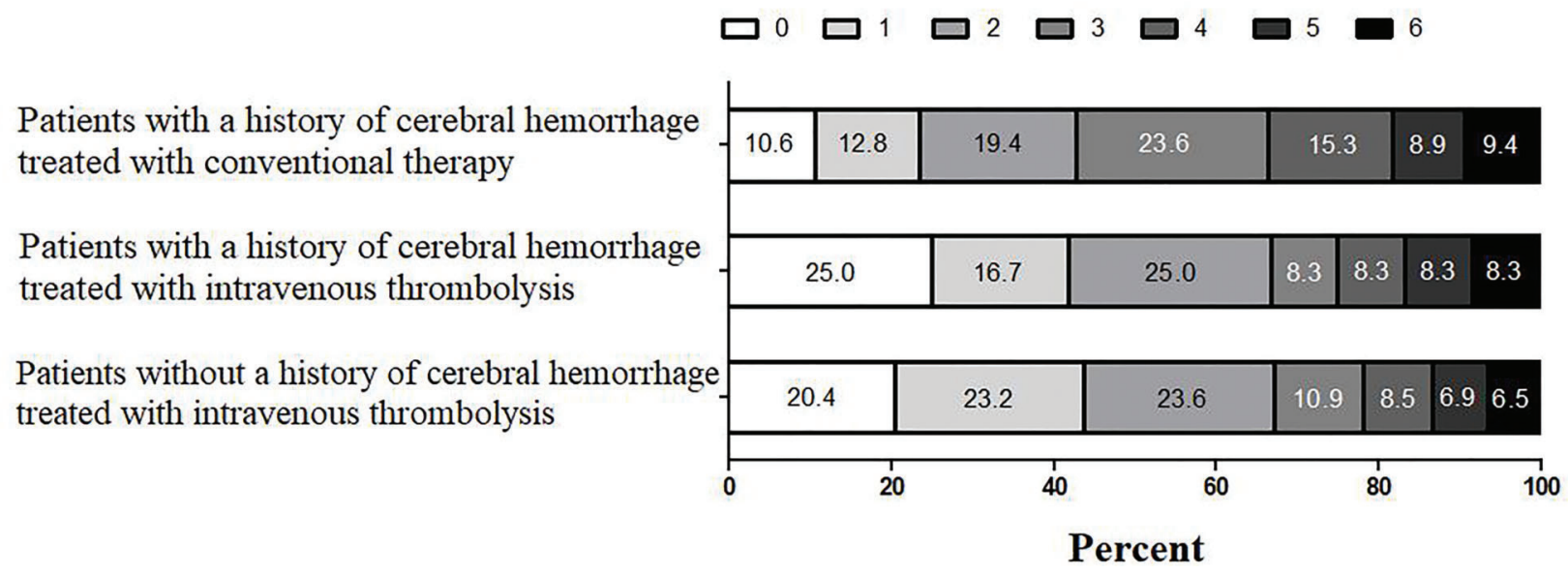

Figure 2. Functional outcomes at 90 days, according to modified Rankin scale (mRS) score (0-6): a score of 0 indicated no symptoms, 1 indicated symptoms without clinically significant disability, 2 indicated slight disability, 3 indicated moderate disability, 4 indicated moderately severe disability, 5 indicated severe disability, and 6 indicated death.

thrombolysis. Prospective, randomized controlled trials with large sample sizes from multiple treatment centers are needed to observe the relationship between incidence of cerebral hemorrhage and the amount of bleeding and the safety of thrombolysis.

\section{Acknowledgments}

This work was supported by grants from the Shandong Provincial Natural Science Foundation (ZR2015HM024) (S-L. Xu). 


\section{References}

1. Prabhakaran S, Ruff I, Bernstein RA. Acute stroke intervention: a systematic review. JAMA 2015; 313: 1451-1462, doi: 10.1001/jama.2015.3058.

2. Sierra C. Essential hypertension, cerebral white matter pathology and ischemic stroke. Curr Med Chem 2014; 21: 2156-2164, doi: 10.2174/0929867321666131227155140.

3. van Asch CJ, Luitse MJ, Rinkel GJ, van der Tweel I, Algra A, Klijn CJ. Incidence, case fatality, and functional outcome of intracerebral haemorrhage over time, according to age, sex, and ethnic origin: a systematic review and meta-analysis. Lancet Neurol 2010; 9: 167-176, doi: 10.1016/S1474-4422 (09)70340-0.

4. Romano JG, Smith EE, Liang L, Gardener H, Camp S, Shuey $L$, et al. Outcomes in mild acute ischemic stroke treated with intravenous thrombolysis: a retrospective analysis of the get with the guidelines-stroke registry. JAMA Neurol 2015; 72: 423-431, doi: 10.1001/jamaneurol.2014.4354.

5. Cronin CA, Shah N, Morovati T, Hermann LD, Sheth KN. No increased risk of symptomatic intracerebral hemorrhage after thrombolysis in patients with European Cooperative Acute Stroke Study (ECASS) exclusion criteria. Stroke 2012; 43: 1684-1686, doi: 10.1161/STROKEAHA.112.656587.

6. Jauch EC, Saver JL, Adams HP Jr, Bruno A, Connors JJ, Demaerschalk BM, et al. Guidelines for the early management of patients with acute ischemic stroke: a guideline for healthcare professionals from the American Heart Association/ American Stroke Association. Stroke 2013; 44: 870-947, doi: 10.1161/STR.0b013e318284056a.

7. Steiner $\mathrm{T}$, Al-Shahi Salman $\mathrm{R}$, Beer $\mathrm{R}$, Christensen $\mathrm{H}$, Cordonnier C, Csiba L, et al. European Stroke Organisation (ESO) guidelines for the management of spontaneous intracerebral hemorrhage. Int J Stroke 2014; 9: 840-855, doi: $10.1111 /$ ijs. 12309 .

8. De Keyser J, Gdovinova Z, Uyttenboogaart M, Vroomen PC, Luijckx GJ. Intravenous alteplase for stroke: beyond the guidelines and in particular clinical situations. Stroke 2007; 38: 2612-2618, doi: 10.1161/STROKEAHA.106.480566.

9. Demaerschalk BM, Kleindorfer DO, Adeoye OM, Demchuk AM, Fugate JE, Grotta JC, et al. Scientific rationale for the inclusion and exclusion criteria for intravenous alteplase in acute ischemic stroke: A statement for healthcare professionals from the American Heart Association/American Stroke Association. Stroke 2016; 47: 581-641, doi: 10.1161/ STR.0000000000000086.

10. Demaerschalk BM. Challenging the validity of imposing contraindications to thrombolysis for acute ischemic stroke. Neurology 2011; 77: 1862-1863, doi: 10.1212/WNL.0b013e 318238 eee8.
11. Tsivgoulis G, Safouris A, Alexandrov AV. Safety of intravenous thrombolysis for acute ischemic stroke in specific conditions. Expert Opin Drug Saf 2015; 14: 845-864, doi: 10.1517/ 14740338.2015.1032242.

12. Klatsky AL, Tran HN. Alcohol and stroke: the splitters win again. BMC Med 2016; 14: 193, doi: 10.1186/s12916-016-0750-z.

13. Passaglia $P$, Ceron CS, Mecawi AS, Antunes-Rodrigues J, Coelho EB, Tirapelli CR. Angiotensin type 1 receptor mediates chronic ethanol consumption-induced hypertension and vascular oxidative stress. Vascul Pharmacol 2015; 74: 49-59, doi: 10.1016/j.vph.2015.04.002.

14. Marchi KC, Muniz JJ, Tirapelli CR. Hypertension and chronic ethanol consumption: What do we know after a century of study? World J Cardiol 2014; 6: 283-294, doi: 10.4330/wjc.v6.i5.283.

15. Lee SH, Kim BJ, Han MK, Park TH, Lee KB, Lee BC, et al. Should we exclude acute stroke patients with previous intracerebral hemorrhage from receiving intravenous thrombolysis? Int J Stroke 2016; 11: 783-790, doi: 10.1177/ 1747493016654289.

16. AbdelRazek MA, Mowla A, Hojnacki D, Zimmer W, Elsadek $\mathrm{R}$, Abdelhamid $\mathrm{N}$, et al. Prior asymptomatic parenchymal hemorrhage does not increase the risk for intracranial hemorrhage after intravenous thrombolysis. Cerebrovasc Dis 2015; 40: 201-204, doi: 10.1159/000439141.

17. Meretoja A, Putaala J, Tatlisumak T, Atula S, Artto V, Curtze $S$, et al. Off-label thrombolysis is not associated with poor outcome in patients with stroke. Stroke 2010; 41: 1450-1458, doi: 10.1161/STROKEAHA.109.576140.

18. Yamaguchi T, Mori E, Minematsu K, Nakagawara J, Hashi K, Saito I, et al. Alteplase at $0.6 \mathrm{mg} / \mathrm{kg}$ for acute ischemic stroke within 3 hours of onset: Japan Alteplase Clinical Trial (J-ACT). Stroke 2006; 37: 1810-1815, doi: 10.1161/01.STR. 0000227191.01792.e3.

19. Mori E, Minematsu K, Nakagawara J, Yamaguchi T, Sasaki $\mathrm{M}$, Hirano T, et al. Effects of $0.6 \mathrm{mg} / \mathrm{kg}$ intravenous alteplase on vascular and clinical outcomes in middle cerebral artery occlusion: Japan Alteplase Clinical Trial II (J-ACT II). Stroke 2010; 41: 461-465, doi: 10.1161/STROKEAHA.109.573477.

20. Nakagawara J, Minematsu K, Okada Y, Tanahashi N, Nagahiro S, Mori E, et al. Thrombolysis with $0.6 \mathrm{mg} / \mathrm{kg}$ intravenous alteplase for acute ischemic stroke in routine clinical practice: the Japan post-Marketing Alteplase Registration Study (J-MARS). Stroke 2010; 41: 1984-1989, doi: 10.1161/ STROKEAHA.110.589606.

21. Anderson CS, Robinson T, Lindley RI, Arima H, Lavados $\mathrm{PM}$, Lee $\mathrm{TH}$, et al. Low-dose versus standard-dose intravenous alteplase in acute ischemic stroke. $N$ Engl $\mathrm{J}$ Med 2016; 374: 2313-2323, doi: 10.1056/NEJMoa1515510. 